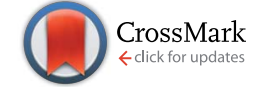

Cite this: RSC Adv., 2017, 7, 17173

\title{
Rapid transformation of transparent conducting films into superhydrophobic conductive films $\dagger$
}

\begin{abstract}
Jong Seok Woo, ${ }^{\text {ab }}$ Geon-Woong Lee, ${ }^{a}$ Soo-Young Park ${ }^{\star b}$ and Joong Tark Han*a
A smart multifunctional surface of conductive plastics with a superhydrophobic surface having porous micro- and nano-structures can potentially be very useful in many applications of electrostatic dissipation (ESD), electromagnetic interference (EMI) shielding, and in transparent film heaters with selfcleaning properties. Here, we demonstrate a facile and rapid method for fabricating superhydrophobic conductive films from transparent conductive films with silver nanowires (AgNWs) and single-walled carbon nanotubes (SWCNTs) on polycarbonate (PC). This process involves the swelling of the PC surface in a dispersion of multi-walled CNTs (MWCNTs) in methyl ethyl ketone (MEK), followed by coagulation in isopropyl alcohol (IPA, nonsolvent for PC). During swelling, the AgNWs and SWCNTs migrated into the plastic, and after that, the swollen PC chains were crystallized in IPA. Notably, by adding MWCNTs in MEK, the crystallization of PC chains was accelerated, and the rapid increase in the electrical resistivity of the film was minimized by reducing the formation of microstructures. Crystallization of the AgNW/ SWCNT electrode onto PC and the incorporation of MWCNTs during crystallization provided a flexible superhydrophobic heater for use as a self-cleaning surface.
\end{abstract}

Received 22nd January 2017 Accepted 14th March 2017

DOI: $10.1039 / \mathrm{c} 7 \mathrm{ra00931c}$

rsc.li/rsc-advances alternative method of transforming conductive films into superhydrophobic surface. Polycarbonate (PC) is widely utilized as an engineering plastic in optical films, and its surface can be crystallized in solution by swelling and coagulation. Previously, many researchers have investigated methods to achieve a superhydrophobic PC surface by controlling the immersion time in a solvent and the coagulation in a non-solvent. ${ }^{20-23}$ However, no attempt has been made to texture a PC surface coated with conducting nanomaterials.

Here, we report that transparent AgNW/SWCNT hybrid films on PC transformed rapidly into superhydrophobic surfaces without deteriorating the electrically conductivity. This process includes immersing the films in a good solvent containing multi-walled CNTs (MWCNTs) for $30 \mathrm{~s}$, followed by immersion in a non-solvent. The crystallization of PC, induced by MWCNTs, was monitored by Raman spectroscopy. The electrical performance of the fabricated films was also demonstrated using a thin films heater and a thermochromic display.

\section{Results and discussion}

Flexible TCFs coated with AgNWs and SWCNTs were prepared by direct air-spraying of aqueous hybrid dispersion of AgNWs and SWCNTs on PC substrates. Fig. 1 shows the scheme for the fabrication of a superhydrophobic and conductive film on the plastic substrate from the AgNW/SWCNT hybrid film by solution crystallization of PC chains. First, aqueous AgNW and SWCNT dispersions were directly mixed at a ratio of 97.2/2.5 (w/ w). This was achieved by functionalizing SWCNTs with 2-uredio-
${ }^{a}$ Nano Hybrid Technology Research Center, Korea Electrotechnology Research Institute, Changwon, 641-120, Republic of Korea.E-mail: jthan@keri.re.kr

${ }^{b}$ Major in Polymer Science and Engineering, School of Applied Chemical Engineering, Kyungpook National University, \#1370 Sangyuk-dong, Buk-gu, Daegu, 702-701, Republic of Korea. E-mail: psy@knu.ac.kr

$\uparrow$ Electronic supplementary information (ESI) available. See DOI: 10.1039/c7ra00931c 
(a)

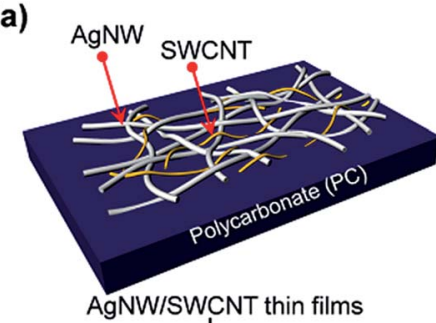

(b)

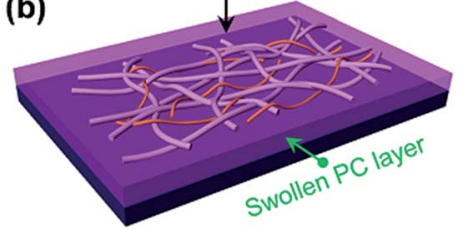

Migration of conducting materials into PC

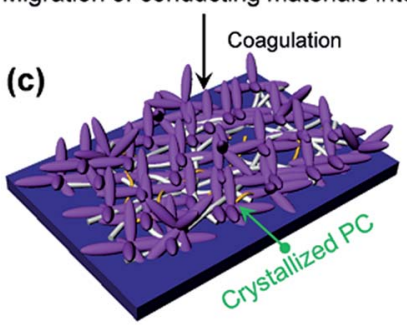

CNT-induced crystallization of PC chains

Fig. 1 Schematics diagram for multifunctional superhydrophobic polymer surface. (a) AgNW/SWCNT hybrid electrodes onto PC substrate of (b) the migration of conducting materials after immersion in MEK/CNTs and (c) CNT-induced crystallization of PC chain, followed by dipping in IPA for several seconds.

$4[1 H]$ pyrimidinone (UHP) groups, which have a high affinity to water molecules. $^{24}$

This solution was air-sprayed with a $\mathrm{N}_{2}$ flow rate of $12 \mathrm{~L}$ $\min ^{-1}$ to fabricate TCFs on the PC substrate. The SWCNT networks can stabilize the AgNWs on hydrophobic PC surfaces. $^{25}$ The AgNW/SWCNT hybrid film shows a low sheet resistance $\left(R_{\mathrm{s}}\right)$ of $15.8 \mathrm{ohm} \mathrm{sq}{ }^{-1}$ at $90.8 \%$ transmittance. The asprepared films were very hydrophilic water contact angle (CA $27^{\circ}$ ) because of the hydrophilic characteristics of AgNWs decorated with a small amount of polyvinylpyrrolidone, which was used as a stabilizer in AgNW synthesis. Thus, we developed a method for transforming this hydrophilic film into a hydrophobic film. Embedding the AgNW/SWCNTs into the plastic substrate can slightly enhance the hydrophobicity, corresponding to the surface energy of the plastic substrate. This facile route of enhancing the hydrophobicity increases the surface roughness, where air can be trapped to minimize the contact area between the solid and the water droplet. To achieve this, AgNW/SWCNT films on PC were immersed in MEK to swell its surface, and subsequently coagulated in IPA as previously reported. ${ }^{26} \mathrm{Fig}$. 2a shows the change in the water CA for varying immersion times in MEK. Immersion for $60 \mathrm{~s}$ triggered the transformation of the PC surface into a superhydrophobic surface with a high water CA, whereas a short immersion time was not enough to increase the water CA on the surface without
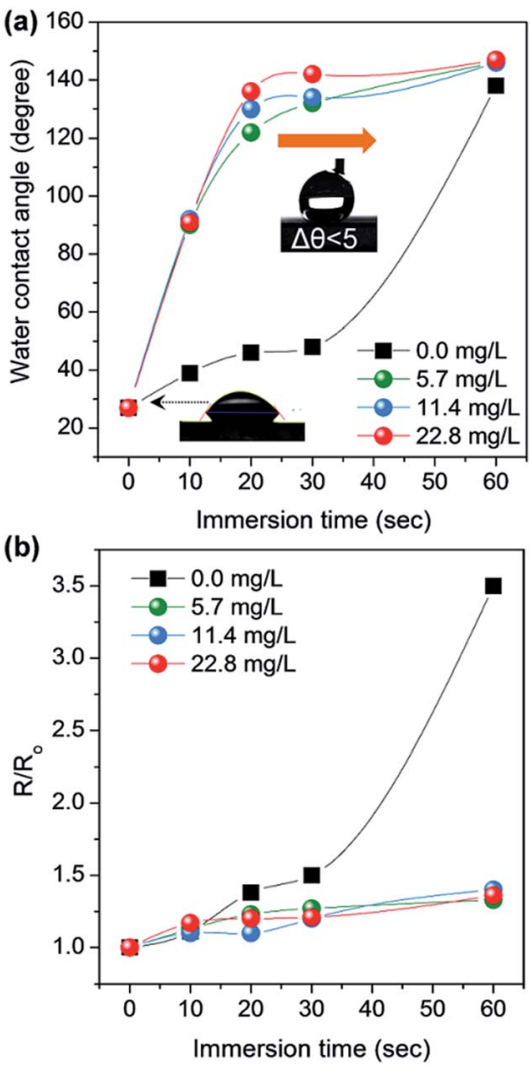

Fig. 2 (a) Water CA and (b) $R_{\mathrm{s}}$ changes after immersion of AgNW/ SWCNT films in MEK and MEK/CNT solution for varying MWCNTs concentration. Lower optical image of a water droplet on as-prepared AgNW/SWCNT film. Upper optical image of a slowly moving water droplet on the PC surface after CNT-induced crystallization of PC.

other additives. Fig. 3 shows the FESEM images of AgNW/ SWCNT films before (Fig. 3a) and after (Fig. 3b-f) immersion by varying the immersion time. After $30 \mathrm{~s}$, crystallized PC structures were observed on the surface. However, even after $30 \mathrm{~s}$, the AgNWs were easily observed on the surface, which triggered the easy wetting of the water droplet. After longer immersion for $60 \mathrm{~s}$, a crystallized hierarchical PC surface with micro- and nano-structures, similar to a lotus leaf, was observed on the AgNW/SWCNT layer, as shown in Fig. 3c, which is a typical superhydrophobic surface morphology. However, the micro-structures on the AgNW/SWCNT layer dramatically increased the $R_{\mathrm{S}}$ of the film, as shown in Fig. 2b. In order to prevent the formation of microstructures, fast crystallization of the swollen PC layer has to be induced by adding additives such as a nucleation agent. Carbon materials are good nucleation agents in the solution crystallization of polymers because of their hydrophobic characteristics, as reported previously. ${ }^{27,28}$ Accordingly, in this study, MWCNTs were dispersed in MEK by varying the concentration in order to investigate the crystallization rate of the swollen PC in IPA. MWCNTs were first dispersed in dimethyl formamide (DMF) at a concentration of $400 \mathrm{mg} \mathrm{L}^{-1}$, which is a favourable solvent for the dispersion of CNTs in terms of the solubility parameter. This MWCNT/DMF solution was directly mixed with MEK. 

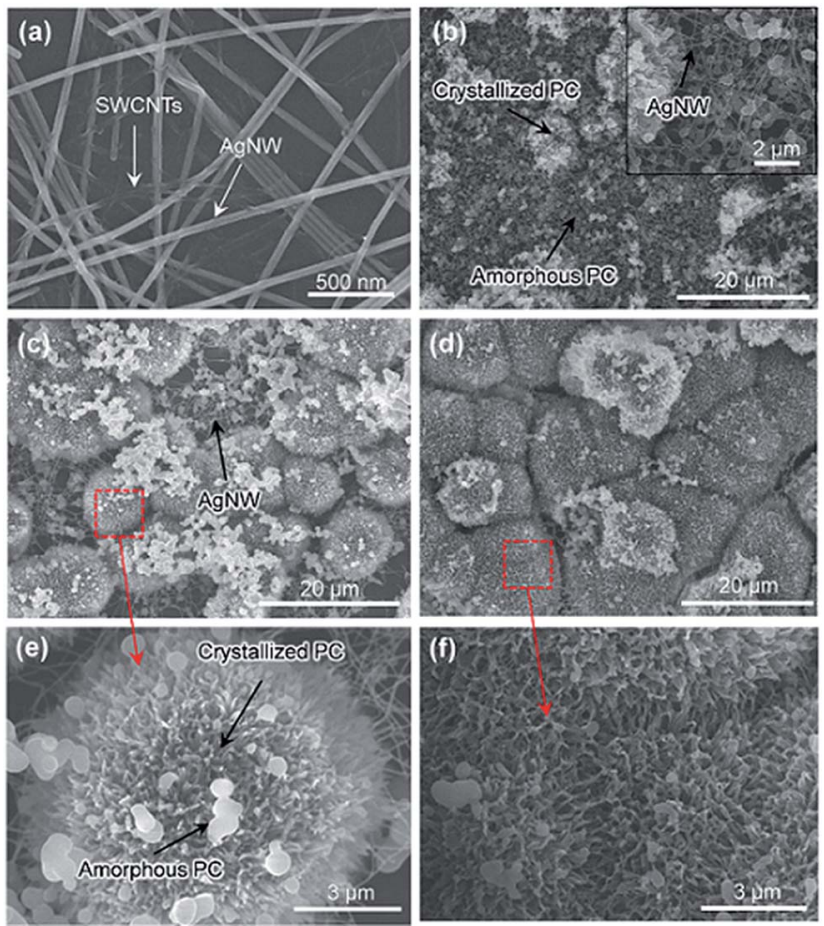

Fig. 3 FESEM images of the (a) pristine AgNW/SWCNT hybrid electrode and PC surface after immersion in MEK for (b) $30 \mathrm{~s}$, (c) $60 \mathrm{~s}$, and (d) $120 \mathrm{~s}$, and subsequent dipping all of samples in IPA for $10 \mathrm{~s}$. High magnification FESEM images of the PC surface after immersion in MEK (e) $60 \mathrm{~s}$, and (f) $120 \mathrm{~s}$.

Fig. 2a shows the water CA changes of the film by varying the concentration of MWCNTs in MEK after coagulation in IPA. On increasing of MWCNT up to $22.8 \mathrm{mg} \mathrm{L}^{-1}$, the AgNW/ SWCNT film was rapidly transformed into a superhydrophobic surface. Notably, the steep increase in the $R_{\mathrm{S}}$ of the film was significantly reduced after addition of MWCNTs in the swelling solvent. Fig. 4 shows the FESEM images of the superhydrophobic surface of the AgNW/SWCNT film after immersion in MWCNT/MEK and coagulation in IPA. The micro-structures on the PC surface disappeared upon adding MWCNTs in the swelling solvent, and nano-structured crystalline PC was formed. This structure change led to decrease in the $R_{\mathrm{S}}$ increases of the film. The presence of MWCNTs in the
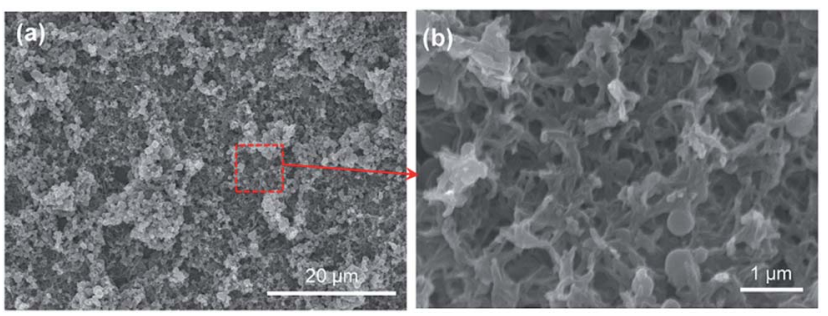

Fig. 4 FESEM images of (a) the PC surface after immersion in the MWCNT/MEK solution containing $22.8 \mathrm{mg} \mathrm{L}^{-1}$ for $30 \mathrm{~s}$, and subsequent dipping it in IPA for $10 \mathrm{~s}$. (b) shows high magnification image of (a). superhydrophobic surface was confirmed by Raman spectroscopy. Fig. 5 shows the Raman spectra of pristine PC and the PC surface crystallized by MWCNTs. The transition from amorphous to crystalline PC was observed in a region corresponding to the stretching vibration of the $\mathrm{C}-\mathrm{O}-\mathrm{C}$ group at $1235 \mathrm{~cm}^{-1}$. The amorphous PC was shifted to $1248 \mathrm{~cm}^{-1}$ after dipping in the MEK and MWCNTs solution. This observation indicated the amorphous PC crystallized upon sequential dipping in the MEK solution and IPA. Notably, even at a MWCNT/MEK concentration of $5.7 \mathrm{mg} \mathrm{L}^{-1}$, the crystalline PC peak was observed after $30 \mathrm{~s}$ dipping, which indicates that a small amount of MWCNTs can work as a nucleation agent. Moreover, the incorporation of MWCNTs was confirmed by the presence of the G-band peaks below $1580 \mathrm{~cm}^{-1}$ and a D-band peak at $1350 \mathrm{~cm}^{-1}$, both because of the SWCNTs and MWCNT. Therefore, it was found that the carbon materials were excellent heterogeneous nucleating agents for solvent crystallization of $\mathrm{PC}$, resulting in a rapid transformation of the $\mathrm{PC}$ surface into the superhydrophobic surface minimizing the increase of the $R_{\mathrm{s}}$.

To demonstrate the superhydrophobic and conductive properties of the prepared film, a thin-film heater was fabricated by applying DC voltage. Fig. 6a shows the heating behaviour of the films as a function of time at applied input voltages. At $7 \mathrm{~V}$, the temperature reached a saturated (steadystate) value of $45{ }^{\circ} \mathrm{C}$. In addition, a thermochromic material was sprayed through shadow mask on the superhydrophobic surface and DC voltage was applied. We then observed the discoloration of the thermochromic material pattern (red colour) after applying DC voltage (Fig. 6b). When a voltage of $7 \mathrm{~V}$ was applied, the red coloured pattern disappeared at $40{ }^{\circ} \mathrm{C}$. These discoloured thermochromic patterns were recovered at $0 \mathrm{~V}$ and the surface was still superhydrophobic (Fig. 6c). These results indicate that our strategy is a promising method of

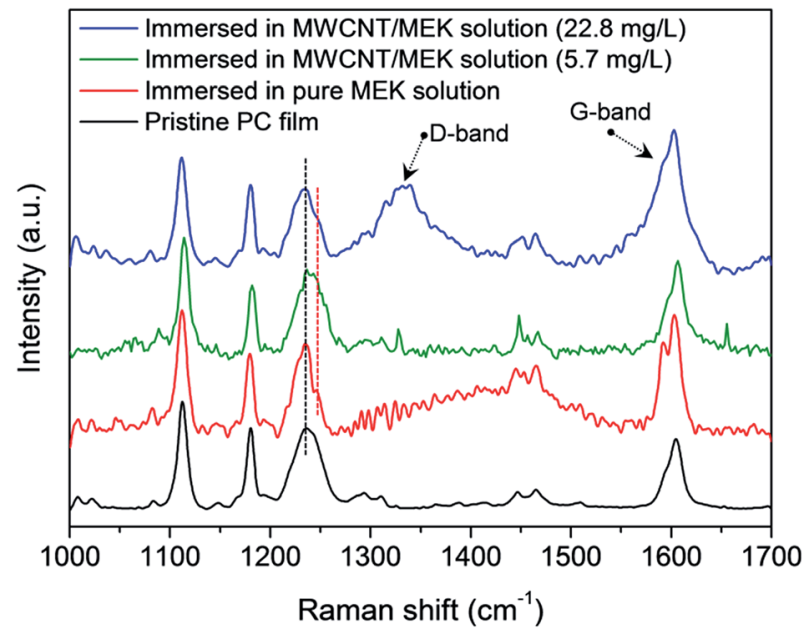

Fig. 5 Raman spectra excited at $633 \mathrm{~nm}$ of pristine $\mathrm{PC}$ and $\mathrm{PC}$ samples immersed in the MEK for $60 \mathrm{~s}$ and the MWCNT/MEK solution for $30 \mathrm{~s}$, and subsequent dipping all of samples in IPA for $10 \mathrm{~s}$. Dotted black and red lines indicate the amorphous and crystalline $P C$, respectively. The dotted blue line indicates the G band from the SWCNTs and MWCNTs. 

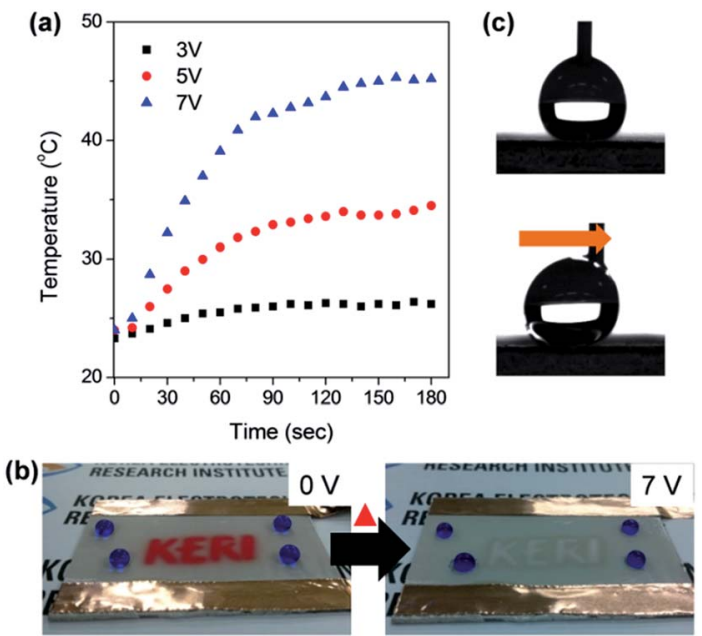

Fig. 6 (a) Heating performance on the conductive and superhydrophobic PC surface. (b) Thermochromic display made with the superhydrophobic surface after increasing the voltage and the water droplet still showed the superhydrophobicity. (c) The static (upper) and dragging (lower) water contact angle images after cooling of the conductive superhydrophobic surface.

rapidly transforming the AgNW/SWCNT film into an electrically conductive and anti-wetting film.

\section{Conclusions}

In summary, we have developed conductive and superhydrophobic PC surfaces with the formation of surface textures, prepared by controlling the crystallization behaviour of PC by solvent-induced crystallization and addition of MWCNTs. The porous nanostructures of the PC crystal were responsible for the superhydrophobicity minimizing the $R_{\mathrm{s}}$ increase of the film by depressing the micro-structure formation with MWCNTs as a nucleation agent. Our results provide a very easy way to fabricate a conductive and superhydrophobic polymer surfaces with lotus-like bionic nanostructures.

\section{Experimental}

\section{Materials}

SWCNTs produced by the arc-discharge method were purchased from Nano Solutions Inc. AgNWs with an average length of $25 \mu \mathrm{m}$ and average diameter of $35 \mathrm{~nm}$, dispersed in water $(0.5 \mathrm{wt} \%)$, were purchased from Nanopixys Inc. MWCNTs were obtained from Hanwha Nanotech Inc.

\section{Preparation of AgNW/SWCNTs hybrid films}

The SWCNTs functionalized with quadruple hydrogen bonding motif pastes $^{6}$ were mixed with the as-received AgNWs suspension to obtain stable AgNW/SWCNT solutions. The AgNW/ SWCNT electrode was fabricated by depositing the prepared solution onto PC substrates using an automatic spray coater (NCS Co. Ltd, NCS-400).
Fabrication of the conductive and superhydrophobic PC surface

MWCNTs were functionalized by applying an ultraviolet (UV)ozone treatment for $1 \mathrm{~h}$. Amorphous PC sheets were obtained from I-Component Corp. and used as received. DMF, MEK, IPA were purchased from Aldrich and used as received. Varying concentrations of MWCNTs $\left(100 \mathrm{mg} \mathrm{L}^{-1}\right.$ to $\left.400 \mathrm{mg} \mathrm{L}^{-1}\right)$ were dispersed in $1 \mathrm{~L}$ of DMF for $1 \mathrm{~h}$ by horn sonication. These MWCNT-dispersed solutions were diluted to investigate the effect of the MWCNT concentration in MEK solution on the crystallization of PC. The immersion time of PC was controlled from 10 to $120 \mathrm{~s}$. AgNW/SWCNT hybrid films on PC were immersed in pure MEK and MWCNT/MEK solution with varying concentrations, and the AgNW/SWCNT films were subsequently immersed in IPA to coagulate or crystallize the swollen PC for $10 \mathrm{~s}$.

\section{Fabrication of the superhydrophobic heating system}

The superhydrophobic heater was fabricated with a twoterminal side contact configuration. A DC voltage was supplied to the superhydrophobic heater, the screen-printed silver paste, and the copper contact at the film edges using a power supply. Superhydrophobic films with an area of $3 \times 7$ $\mathrm{cm}^{2}$ were fabricated.

\section{Characterization}

The $R_{\mathrm{S}}$ of the film was measured using a four-probe tester (Loresta MCP-T610). The transmittance of the film was measured using an ultraviolet-visible-near-infrared (UV-vis-NIR) spectrometer (Varian, Cary 500). The water CA was measured using a contact angle analyser (SEO, Phoenix 300). The corresponding images of the resulting thin film were obtained using a field emission scanning microscope (Hitachi, S4800). Raman spectra were measured to characterize the electronic structure of the $1 \mathrm{D}$ nanomaterial at $25{ }^{\circ} \mathrm{C}$, using a high-resolution Raman spectrometer (HORIBA Jobin Yvon, US/HR-800) with an excitation wavelength of $633 \mathrm{~nm}$. The temperature profile of each film was measured using an IR thermal imager (NEC San-ei Instruments, Ltd, TH9100) and a thermocouple (Center Technology Corp., CENTER 300).

\section{Acknowledgements}

This work was supported by the Center for Advanced SoftElectronics funded by the Ministry of Science, ICT and Future Planning as Global Frontier Project (2014M3A6A5060953), and by the Primary Research Program (17-12-N0101-32) of the Korea Electrotechnology Research Institute.

\section{References}

1 Z. C. Wu, Z. H. Chen, X. Du, J. M. Logan, J. Sippel, M. Nikolou, K. Kamaras, J. R. Reynolds, D. B. Tanner, A. F. Hebard and A. G. Rinzeler, Science, 2004, 305, 1273.

2 D. Zhang, K. Ryu, X. Liu, E. Polikarpov, J. Ly, M. E. Tompson and C. Zhou, Nano Lett., 2006, 6, 1880. 
3 L. Hu, D. S. Hecht and G. Grüner, Nano Lett., 2004, 4, 2531.

4 H.-Z. Hong, K. K. Kim, K. P. So, Y. S. Chang and Y. H. Lee, J. Am. Chem. Soc., 2007, 129, 7758.

5 Y. Zhou, L. Hu and G. Grüner, Appl. Phys. Lett., 2006, 88, 123109.

6 J. S. Woo, J. T. Han, S. Jung, J. J. Jang, H. Y. Kim, H. J. Jeong, S. Y. Jeong, K.-J. Baeg and G.-W. Lee, Sci. Rep., 2014, 4, 4804.

7 J. Wang, J. Zhang, A. K. Sundramoorthy, P. Chen and M. B. Chan-Park, Nanoscale, 2014, 6, 4560.

8 B. Liu, C. Li, Q.-L. Liu, J. Dong, C.-W. Guo, H. Wu, H.-Y. Zhou, X.-J. Fan, X. Guo, C. Wang, X.-M. Sun, Y.-H. Jin, Q.-Q. Li and S.-S. Fan, Appl. Phys. Lett., 2015, 106, 033101.

9 Y. Ahn, Y. Jeong, D. Lee and Y. Lee, ACS Nano, 2015, 9, 3125. 10 A. Kumar and C. Zhou, ACS Nano, 2010, 4, 11.

11 J. Zou, H. Chen, A. Chunder, Y. Yu, Q. Huo and L. Zhai, Adv. Mater., 2008, 20, 3337.

12 M. Qu, G. Zhao, X. Cao and J. Zhang, Langmuir, 2008, 24, 4185.

13 A. Das, T. M. Schutzius, S. Byer and C. M. Megaridis, Carbon, 2012, 50, 1346.

14 Y. Zhu, J. Zhang, Y. Zheng, Z. Huang, L. Feng and L. Jiang, Adv. Funct. Mater., 2006, 16, 568.

15 J. T. Han, S. Y. Kim, J. S. Woo and G.-W. Lee, Adv. Mater., 2008, 20, 3724.

16 H. Yao, C.-C. Chu, H.-J. Sue and R. Nishimura, Carbon, 2013, 53,366 .
17 W. Wang, X. Xie and X. Ye, Carbon, 2010, 48, 1670.

18 P. Cataldi, I. S. Bayer, R. Cingolani, S. Marras, R. Chellali and

A. Athanassiou, Sci. Rep., 2016, 6, 27984.

19 D. Dunand, T. Darmanin and F. Guittard, ChemPhysChem, 2013, 14, 2947.

20 N. Zhao, L. Weng, X. Zhang, Q. Xie, X. Zhang and J. Xu, ChemPhysChem, 2006, 7, 824.

21 N. Zhao, J. Xu, Q. Xie, L. Weng, X. Guo, X. Zhang and L. Shi, Macromol. Rapid Commun., 2005, 26, 1075.

22 H. Y. Erbil, A. L. Demirel, Y. Avci and O. Mert, Science, 2003, 299, 1377.

23 X. Lu, C. Zhang and Y. Han, Macromol. Rapid Commun., 2004, 25, 1606.

24 J. T. Han, B. H. Jeong, S. H. Seo, K. C. Roh, S. Kim, S. Choi, J. S. Woo, H. Y. Kim, J. I. Jang, D.-C. Shin, S. Jeong, H. J. Jeong, S. Y. Jeong and G.-W. Lee, Nat. Commun., 2013, 4, 2941.

25 J. S. Woo, B. K. Kim, H. Y. Kim, G.-W. Lee, S.-Y. Park and J. T. Han, RSC Adv., 2016, 6, 86395.

26 J. T. Han, J. S. Kim, S. H. Kim, H. S. Lim, H. J. JEong, S. Y. Jeong and G.-W. Lee, ACS Appl. Mater. Interfaces, 2010, 2, 3379 .

27 J. Y. Kim, H. S. Park and S. H. Kim, Polymer, 2006, 47, 1379.

28 K. Okada, K. Watanabe, T. Urushihara, A. Toda and M. Hikosaka, Polymer, 2007, 48, 401. 